\title{
Optoelectronic Device for Measuring the Power of Laser Radiation
}

\author{
Dimcho Pulov \\ Department of Mechanical and Precision Engineering \\ Technical University of Gabrovo \\ Gabrovo, Bulgaria \\ pulov@mail.bg
}

\begin{abstract}
A device for measuring the power of the laser radiation.has been designed. The device consists of transmitter and receiving part. The transmitter includes optical radiation source and optical system for collimation of radiation. The receiver part consists of silicon photodiodes, electronic signal processing unit and unit for measurements.
\end{abstract}

Keywords - Gaussian laser beam; Galilean beam expander; receiver the laser source; photodiodes

\section{INTRODUCTION}

Lasers are monochromatic coherent sources of optical radiation characterized by a low degree of divergence and high intensity. These properties determine their wide application in different fields. The power of the laser radiation is of great importance when used for practical purposes. This requires development of new methods and devices for measuring and reducing the power of laser radiation.

\section{EXPOSITION}

The purpose of this article is to develop a device for measuring the power of high-energy and highly concentrated optical fluxes. Such fluxes are formed during the generation of laser radiation.

The set goal can be achieved by accomplishing the following tasks:

- Development of a block functional diagram of the device;

- Development of the optical diagram of laser beam expander;

- Development schematic electrical diagram of the device.

- Development of the algorithm of the control program

\author{
Tsanko Karadzhov \\ Department of Mechanical and Precision Engineering \\ Technical University of Gabrovo \\ Gabrovo, Bulgaria \\ karadjov_s@abv.bg
}

\section{A. Spatial profile of the laser beam}

The design of the optical laser systems to a certain extent repeats that of the classic optical devices but taking into account the features of laser radiation [5], [8], [18]. Such a feature is the spatial structure of the laser beam. In its consideration the concept of Gaussian beam is of fundamental importance. This is a beam in the cross section of which the energy is distributed according to Gauss's law (Fig. 1). The Gaussian beam corresponds to the fundamental mode $T E M_{00}$ which is formed by a stable resonator with an arbitrary configuration. Its intensity in the cross section is determined by the expression

$$
I(r)=I(0) \cdot e^{-2\left(\frac{r}{\omega}\right)^{2}}=\frac{2 P}{\pi[\omega(z)]^{2}} \cdot e^{-2\left(\frac{r}{\omega}\right)^{2}}
$$

where: $r$ is a radial distance from the axis of the beam; $I(r)$ and $I(0)$ are the intensity of the beam at distance $r$ and the axis ; $\omega$ is beam radius at which the intensity decreases $e^{2}$ times; $P$ is the full power of the beam

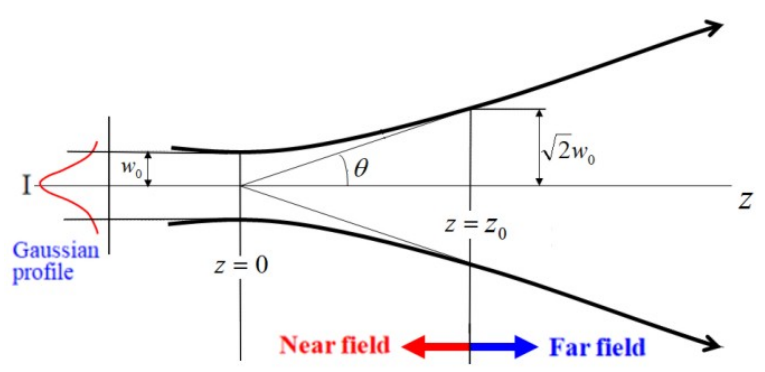

Fig. 1. Spatial profile of the Gaussian laser beam 
Laser beams are coherent and their propagation in space is described by the laws of diffraction. Depending on the longitudinal distance $z$ there is Fresnel diffraction (near zone) and Fraunhofer diffraction (far zone). The Rayleigh range $\left(z=z_{0}\right)$ is taken as the notional line between the two zones. The Rayleigh range represents the distance from the waist of the beam at which its radius increases $\sqrt{2}$ times, and the area of its cross section -2 times.

In the near zone Gaussian beam will at first get closer to the axis and reach a minimum cross section in the waist of the beam, where its radius is

$$
\omega_{0}=\omega(z=0)=\sqrt{\frac{\lambda z_{0}}{\pi}}
$$

After the waist the Gaussian beam undergoes a diffraction divergence and moves away from the axis. Its cross section increases and in the far zone at distance $z$ from the waist the beam radius assumes the value

$$
\omega_{z}=\omega_{0} \sqrt{1+\left(\frac{z}{z_{0}}\right)^{2}}
$$

The wave front of the oscillations occurring in the resonator in the paraxial region is spherical. The radius of the wave front depends on the longitudinal coordinate $Z$ and is determined by the expression

$$
R_{\mathrm{z}}=z\left[1+\left(\frac{\mathrm{z}_{0}}{\mathrm{z}}\right)^{2}\right]
$$

It follows from (1) that at a distance $z=z_{0}$ the radius of the wave front is minimal and has a value $R_{z}=2 z_{0}$. This corresponds to a spherical wave front. As $z$ decreases and increases the radius $R_{z}$ increases. $R_{z}$ increases faster when $z$ decreases. The wave front is flat in the waist of the beam $(z=0)$.

The spatial structure of the Gaussian beam during its propagation in space for level $1 / e^{2}$ is given by hyperbolic isophotes. The asymptotes of the enveloping hyperbolas make an angle $\theta$ with the axis of the beam. This angle is equal to the angle of diffraction of the fundamental mode TEM $_{00}$ in the far zone. The angle $\theta$ describes the divergence of the beam in the far zone $\left(z>>z_{0}\right)$ and can be expressed as

$$
\theta=\frac{\lambda}{\pi \omega_{0}}
$$

The full divergence of the beam is $\Theta=2 \theta$.
It follows from expression (2) that the smaller the waist of the beam the greater the divergence of the beam. Therefore, to form a well collimated beam, it is necessary to reduce its divergence at the expense of increasing its cross section.

\section{B. Block functional diagram of the device}

The optoelectronic device for measuring the power of laser radiation consists of a transmitting and receiving part [9], [11], [16], [17]. The transmitting part includes laser source and collimating optical system. A laser is used as radiation source. The collimating system is an afocal optical system which acts as a beam expander while reducing its angular divergence. The receiving unit consists of silicon photo receivers, an electronic processing unit and a unit for visualization of measurement result. Fig. 2 shows a block chart of the device.

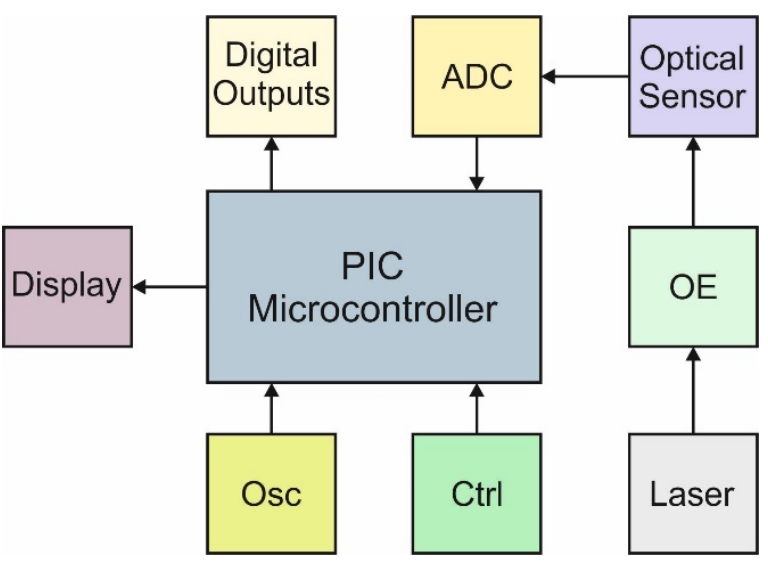

Fig. 2. Block chart of an optoelectronic device for measuring the power of laser radiation

\section{Beam expander}

When designing the optical system (OS) of the laser, it is assumed that the source of radiation is at infinity and its angular dimensions are determined by the divergence of the beam [3], [4], [12], [13], [14]. In order to achieve optimal coordination between the laser and the optical system it is necessary to place the entrance pupil of the optical system in the area of the beam waist [1], [7], [10], [15].

The main task of the OS is to expand the input beam by some coefficient $M$ defined by the ratio of the diameters of the beam waist after the expander and before the expander. This can be achieved by reducing the divergence of the output beam. Such conversion of the laser beam can be achieved by using telescopic afocal reversing system. There are two types of such systems: Galilean and Keplerian. Galilean system was chosen on the basis of the following advantages:

a) Lack of internal focus. This avoids the production of high energy densities, which is important when using high power sources.

b) The first element in Galilean system is negative and it has a positive spherical aberration. The second element is positive and its spherical aberration is negative. Thus, with correct selection of optical forces and shape of 
the elements, it is possible to easily obtain a system with corrected spherical aberration.

\section{c) Galilean system is more compact.}

Propagation of a Gaussian beam through an Galilean expander is shown in Fig. 3. The first element (Lens 1) focuses the beam in its focal plane. Its purpose is to reduce the cross section of the beam waist. In Galilean system, the first element is negative and therefore the waist after it is virtual and located on the left.

The divergence of the beam before lens 1 is determined by expression (2). The radius of the waist after the first element is

$$
\omega_{0}^{\prime}=\frac{\lambda}{\pi \omega_{0}} \cdot f_{1}^{\prime}
$$

The divergence of the beam after the first lens is

$$
\theta^{\prime}=\frac{\lambda}{\pi \omega_{0}^{\prime}}
$$

After lens 1 the beam increases its divergence. The purpose of the second element (lens 2) is to reduce the divergence and to collimate the beam radiation. For this reason, the beam waist after lens 1 is located in the rear focal plane of lens 2 . The focal distance $f^{\prime}{ }_{2}$ of lens 2 is selected from the required expansion $M$ and the degree of beam collimation to be achieved. The greater the $f^{\prime}{ }_{2}$ the greater the beam expansion and the smaller the divergence. The beam waist after lens 2 is determined by the expression

$$
\omega^{\prime \prime}{ }_{0}=\frac{\lambda}{\pi \omega_{0}^{\prime}} \cdot f^{\prime}{ }_{2}
$$

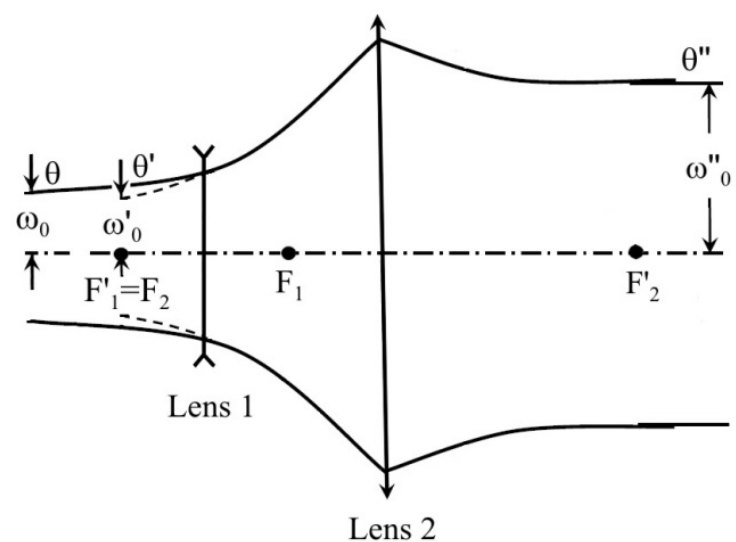

Fig. 3. Propagation of a Gaussian beam through an Galilean expander

The beam divergence after the second lens is

$$
\theta^{\prime \prime}=\frac{\lambda}{\pi \omega^{\prime \prime}{ }_{0}}
$$

Therefore the coefficient of expansion can be written as follows:

$$
M=\frac{\omega_{0}^{\prime \prime}}{\omega_{0}}=\frac{f_{2}^{\prime}}{f_{1}^{\prime}}=\frac{\theta}{\theta^{\prime \prime}}
$$

Fig. 4 shows the optical diagram of the designed beam expander, and Table 1 shows its design parameters. The expander has an magnification $M=5^{x}$, his diameter of the entrance pupil is $3 \mathrm{~mm}$ and diameter of the exit pupil is 15 $\mathrm{mm}$.

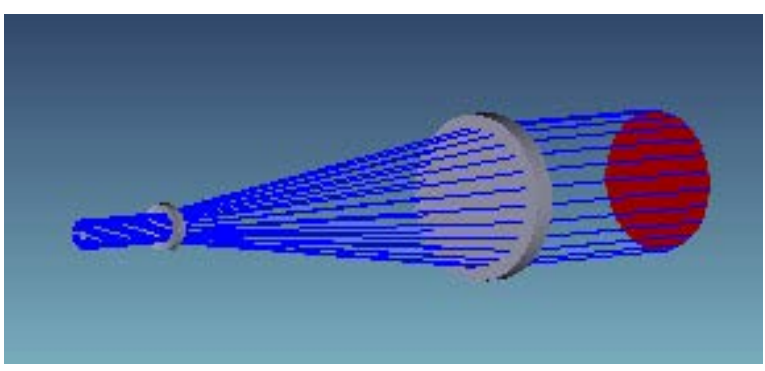

Fig. 4. Optical scheme of the Galilean expander

TABLE 1 GALILEAN EXPANDER DESIGN PARAMETERS

\begin{tabular}{|l|l|l|l|l|}
\hline \multicolumn{2}{|c|}{ Surface } & \multicolumn{1}{c|}{ Radius } & Thickness & Glass \\
\hline \multirow{2}{*}{ Lens 1 } & $\mathbf{1}$ & $-4,4$ & 0,76 & N-SF8 \\
\cline { 2 - 5 } & $\mathbf{2}$ & $-8,45$ & 48,64 & \\
\hline \multirow{2}{*}{ Lens 2} & $\mathbf{3}$ & $\infty$ & 3,35 & N-SF8 \\
\cline { 2 - 5 } & $\mathbf{4}$ & $-44,72$ & & \\
\hline
\end{tabular}

The optical system of the designed expander does not form images of complex objects, but only transmits the laser radiation energy. Therefore, the main requirements for its quality are not related to transmitting fine structure of the image, but to the implementation of the necessary expansion and minimization of the divergence of the output laser beam.

The Marechal criterion $W_{R M S}>\frac{\lambda}{14}$ is used to evaluate the quality of the expander. The RMS value of the wave aberration at the output of the expander is $W_{R M S}=0,007 \lambda$ . Therefore, it can be assumed that it is of diffractionlimited quality. In Fig. 5 the OPD graph is shown at the output of the expander.

\section{Schematic electrical diagram of the device}

Fig. 6 shows the schematic electrical diagram of the device. Precision high speed TSL254 light-to-voltage converters are used as photo receivers.

These converters consist of silicon photodiode with photosensitive area $1 \mathrm{~mm}$ and high impedance operational amplifier with $1 M \Omega$ feedback resistor. The output voltage is proportional to the illuminance. Fig. 7 shows the diagram of the photo converter. The sensitivity of the sensor is $60 \mathrm{mV} / \mu \mathrm{W} / \mathrm{cm}^{2}$ at $\lambda=880 \mathrm{~nm}$. 


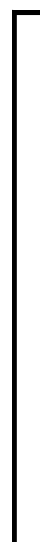

Fig. 5. OPD graph of the expander

An 8 bit PIC18F452 microcontroller is used to control the operation of the device, which has a 10 bit analog-to digital converter. The threshold voltages that determine the measuring range of the analog-to-digital converter are $V_{R E F}=0 \mathrm{~V}$ and $V_{R E F+}=5,12 \mathrm{~V}$ respectively and are applied to terminals RA2 and RA3. The input analog signal that enters the ADC is represented by 210 or 1024 levels. The step of voltage increase is determined by the formula:

$$
\Delta U=\frac{U_{R E F+}}{2^{10}}=\frac{5,12}{1024}=5 \mathrm{mV}
$$

Four photo converters are included in the functional diagram of the device. The measured power is proportional to the average voltage of the photo converters. The voltages at the output of the photo converters are determined when calibrating the device - which values of the laser radiation power they correspond to and accordingly to be entered as correction coefficients in the control program $[2,6]$.

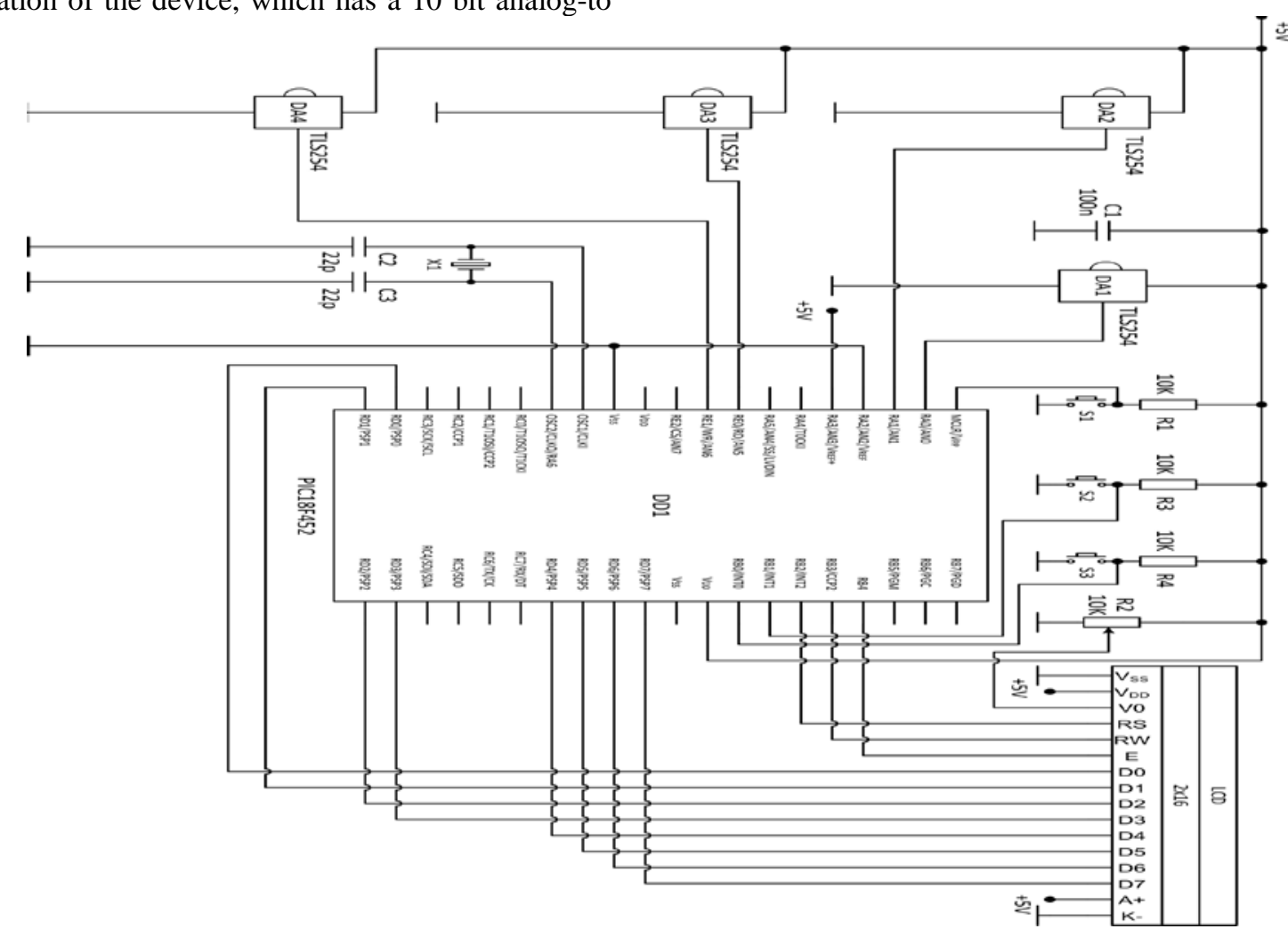

Fig. 6. Schematic electrical diagram of the device

\section{E. Block chart of the algorithm of the program}

The block chart of the algorithm of the control program is shown in fig. 8. At the beginning, the controller is initialized, constants and variables are defined and the corresponding cells from data memory are reserved for them. The next stage of the program is related to determining the input/output ports used. The pins of the microcontroller that will be digital inputs, analog inputs and digital outputs respectively are defined. PIC18F452 has 4 internal timer modules.

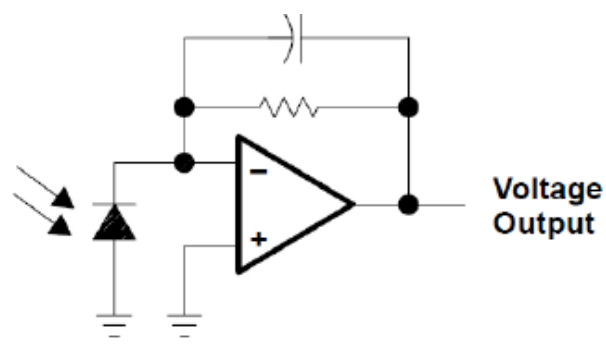

Fig. 7. Diagram of the photo converter 


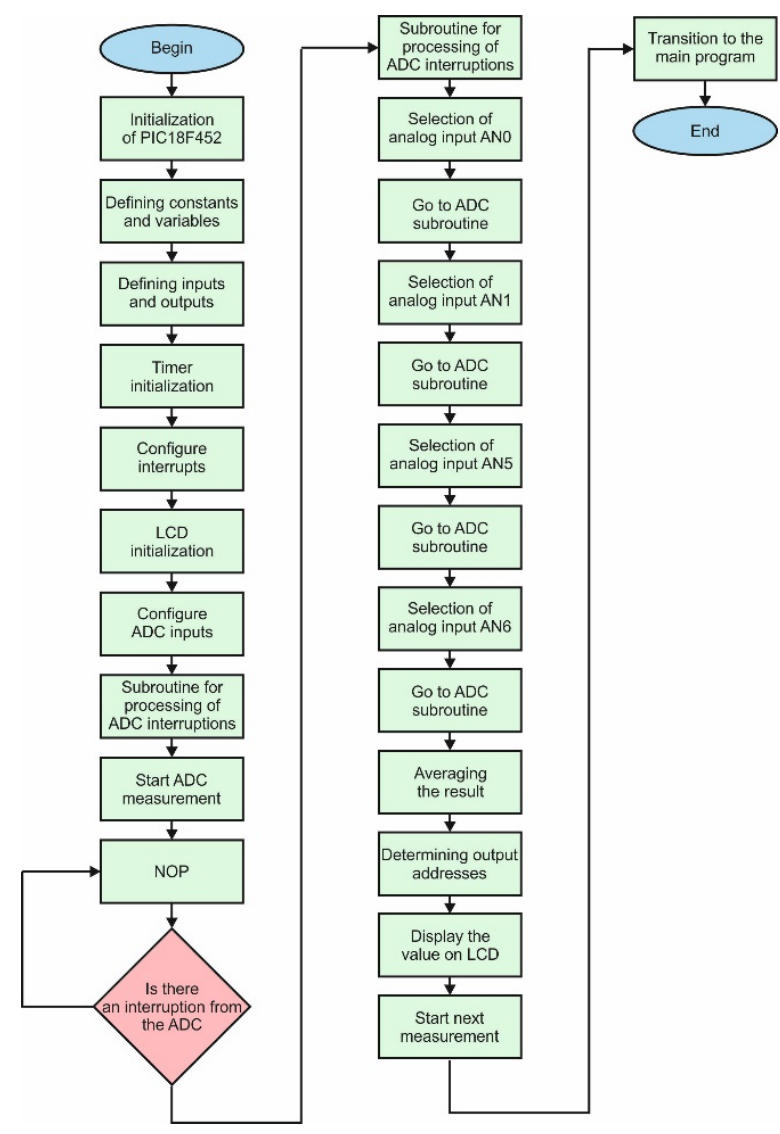

Fig. 8. Block chart of the algorithm of the program

Only Timer 1 is used. It sets time delays in the program which are used to synchronize the operation of the microcontroller and the alphanumeric liquid crystal display. Next, an initialization of the display is performed. The main program is a continuous cycle which stops only when the voltage measurement from the ADC has been completed. The processing of the result of each measurement takes place in a subroutine which starts when an ADC interrupt occurs.

\section{CONCLUSION}

Optoelectronic device for measuring laser radiation power has been developed. $5^{x}$ Galilean beam expander which has a low divergence has been designed. A scheme with four photo receivers has been used, which increases the measurement accuracy compared to a single photo receiver.

\section{REFERENCES}

[1] G. I. Cukanova,A. V. Baholdin, Specialnie razdeli prikladnoj optiki,SPb,GUITMO, 2007.
[2] D. Dichev, H. Koev, T. Bakalova and P. Louda, "A Measuring Method for Gyro-Free Determination of the Parameters of Moving Objects”, Metrology and Measurement Systems, Vol. 23, Issue 1,2016, pp. 107-118, DOI: 10.1515/mms-2016-0001.

[3] H.J. Eichler, J. Eichler and O. Lux, Lasers: Basics, Advances and Applications, Springer Series in Optical Sciences, Springer Nature Switzerland AG, 2018.

[4] I.A. Konyakhin, A. Merson, A.N. Timofeev and A.I. Konyakhin , „Optoelectronic system on the base of the anamorphic element for the measuring of the elevation angles", in Proc. of SPIE, Sixth International Symposium on Precision Engineering Measurements and Instrumentation 2010, Vol. 7544, p. 75443N, DOI: 10.1117/12.885594.

[5] L. Lazov, N. Angelov, E. Teirumnieks, Method for Preliminary Estimation of the Critical Power Density in Laser Technological Processes, Proceedings of the 12th International Scientific and Practical Conference, Rezekne, Latvia, 2019, Vol. 3, pp. 129 -133, DOI: 10.17770/etr2019vol3.4140.

[6] N. Kenarov, PIC mikrokontroleri - chast 2,Mlad konstruktor, Varna, 2006.

[7] Siegman, A.E., Lasers, Oxford University Press, University Science Books, Oxford, England, 1986.

[8] Yu. M. Klimkov, Prikladnaya lazernaya optika, Moskva, Mashinostroenie, 1985

[9] D. Dichev, H. Koev, T. Bakalova and P. Louda, "A Gyro-Free System for Measuring the Parameters of Moving Objects", Measurement Science Review, Vol. 14, Issue 5,2014, pp. 263-269, DOI:10.2478/msr-2014-0036.

[10] Pencheva T., D. Pulov, B. Gyoch, M. Nenkov Design of CCD Optical System for Thermal IR Spectral Region. In pr. 29-th International Spring Seminar fn Electronics Technology, St. Marienthal, Germany, Verlag Dr. Markus A. Detert, 2006, pp. 413418, DOI: 10.1109/ISSE.2006.365380.

[11] D. Shafer, "Laser beam steerer-expander", Applied Optics, Vol. 17, Issue 22, 1978, pp. 3584-3586, DOI: 10.1364/AO.17.003584

[12] M. Scaggs and G. Haas, "Optical alignment influenced aberrations in laser beam delivery systems and their correction", Proc. SPIE 9343, Laser Resonators, Microresonators, and Beam Control XVII, 93430T (March 3, 2015), DOI: 10.1117/12.2076630.

[13] G. Simonova and V. Maximov, „Collimation system design for multi-wavenlength Nd:Yag laser”, Interexpo Geo - Siberia, Vol. 5, Issue 3, 2013, pp.63-67.

[14] G. Kohanenko, M. Makogon, Y. Ponomarev, O. Rinkov and G. Simonova, „Calculation of two-wavelength laser beam expander fluorescent lidar”, Opticheski zhurnal, Vol. 4, Issue 79, 2012, pp. 28-32.

[15] M. Guo, G. Jin, J. Cai, W. Zhang and Z. Wei, "Study and design of beam expander with wide aperture", Proc. SPIE 9295, International Symposium on Optoelectronic Technology and Application 2014: Laser Materials Processing; and Micro/Nano Technologies, 92950T (December 18, 2014).

[16] Sidney A. Self, "Focusing of spherical Gaussian beams", Applied Optics, Vol. 22, Issue 5, 1983, pp. 658-661, DOI: 10.1364/AO.22.000658

[17] H. Sun, "Thin Lens Equation for a Real Laser Beam with Weak Lens Aperture Truncation”, Optical Engineering, Vol. 37, No. 11, 1998, pp. 2903-2913, DOI: 10.1117/1.601877.

[18] D. L. Shealy, "Optical design of laser beam shaping systems," in International Optical Design Conference, 2002 OSA Technical Digest Series (Optical Society of America, 2002), paper IWA2, DOI: 10.1364/iodc.2002.iwa2 